\title{
Gestes et « archéologie du présent » au Japon des années 1920 à aujourd'hui
}

Quelques enseignements pour l'ethnologie francophone

Gestures and Japanese Modernology from the 1920's Onwards: Some Lessons for

French Anthropology

\section{Damien Kunik}

\section{OpenEdition}

Journals

Édition électronique

URL : https://journals.openedition.org/tc/8306

DOI : $10.4000 /$ tc. 8306

ISSN : 1952-420X

Éditeur

Éditions de l'EHESS

\section{Édition imprimée}

Date de publication : 1 décembre 2014

Pagination : 68-83

ISBN : 978-2-7351-2346-9

ISSN : 0248-6016

\section{Référence électronique}

Damien Kunik, « Gestes et « archéologie du présent » au Japon des années 1920 à aujourd'hui », Techniques \& Culture [En ligne], 62 | 2014, mis en ligne le 01 décembre 2017, consulté le 30 septembre 2022. URL : http://journals.openedition.org/tc/8306 ; DOI : https://doi.org/10.4000/tc.8306 


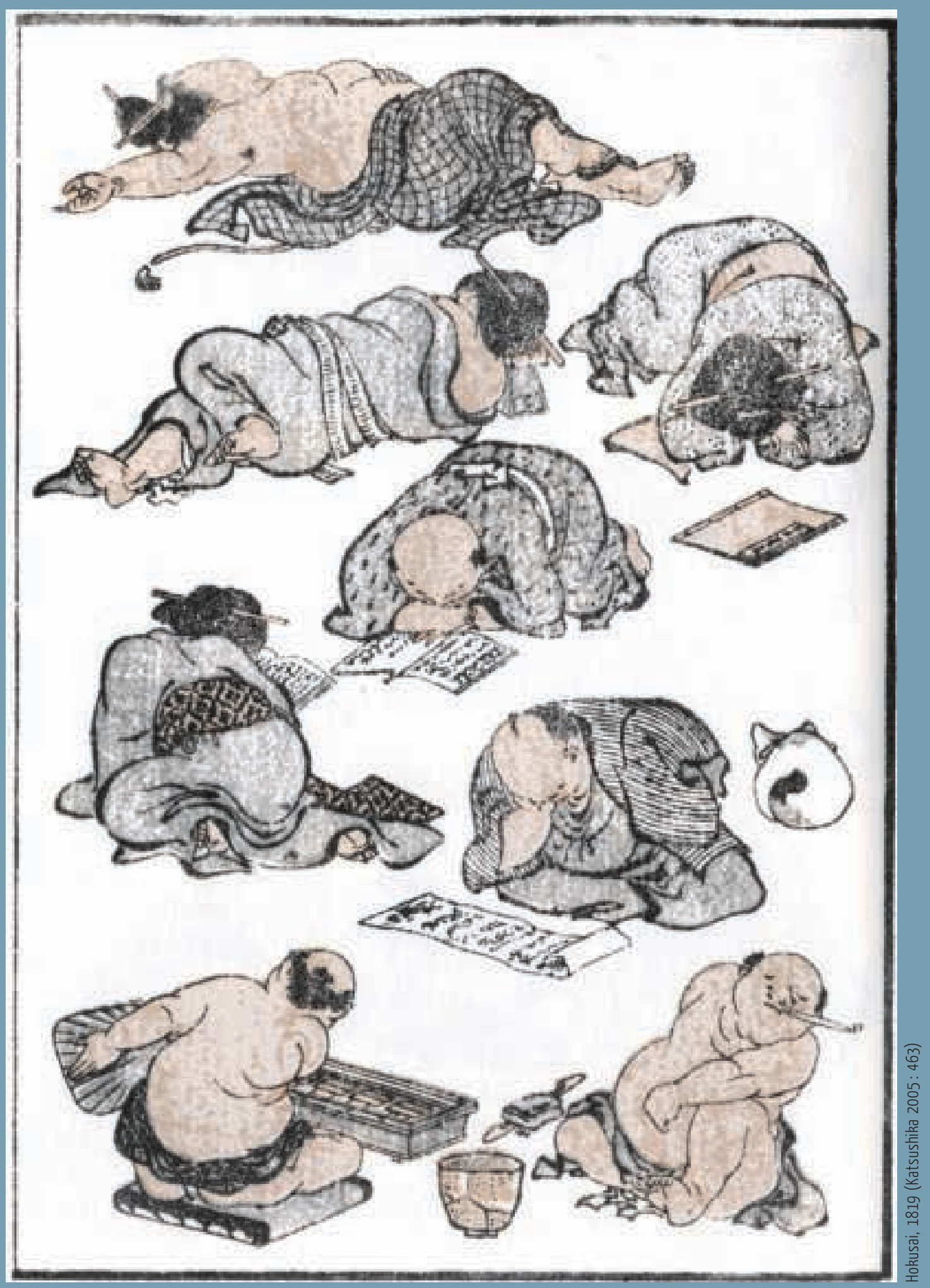




\section{GESTES ET «ARCHÉOLOGIE DU PRÉSENT» AU JAPON DES ANNÉES 1920 À AUJOURD'HUI}

\section{Quelques enseignements pour l'ethnologie francophone}

Le $1^{\text {er }}$ septembre 1923, une secousse sismique d'une intensité rare et les incendies qui en résultent réduisent à néant la ville de Tokyo et sa région. Dans ce contexte stupéfiant, l'architecte Kon Wajirô (1888-1973) va tout d'abord s'intéresser aux initiatives autogérées, individuelles ou collectives, de reconstruction de la métropole. Les premières enquêtes lui font cependant entrevoir des champs de savoir insoupçonnés et rapidement, la seule question architecturale est délaissée au profit d'investigations holistiques de terrain. C'est à la suite du tremblement de terre de 1923 qu'il conçoit ainsi la nature originale de son propos, à mi-chemin entre discours scientifique affirmé et pratique artistique inséparable de son traitement graphique.

Kon est également ethnographe, actif au sein d'une jeune discipline qui se pratique encore essentiellement en amateur. Il est proche des grandes figures du milieu avec lesquelles il se consacre à l'étude de la culture populaire japonaise. On peut compter parmi celles-ci le folkloriste Yanagita Kunio (1875-1962) dont il est un assistant. Il aura également un ascendant intellectuel important sur Shibusawa Keizô (1896-1963), futur fondateur du Musée national d'ethnologie (Kokuritsu minzokugaku hakubutsukan).

Un aspect spécifique du travail de Kon nous préoccupera plus particulièrement ici. L'observation des populations urbaines dans les ruines et le dénuement qui les afflige offre à Kon une opportunité unique de s'intéresser de très près aux pratiques corporelles de ses contemporains, le corps étant compris chez lui aussi comme le «premier et plus naturel instrument de l'homme» (Mauss 1935: I, 10).

Chez Kon, l'étude du corps et du geste s'inscrit tout à la fois dans une longue tradition de représentation graphique de ces questions, initiée dans les rouleaux peints de l'époque 
féodale et rendue célèbre notamment par les croquis de l'illustrateur Katsushika Hokusai (1760-1849). Le travail de Kon peut être envisagé comme une manifestation moderne d'un goût savant pour les typologies visuelles de ce que Mauss appellera bientôt les «techniques du corps». À cette dimension esthétique, le travail de Kon ajoute cependant une vraie portée anthropologique qui doit être comprise comme le premier moment du rapport entre dessin et sciences humaines au sein de l'ethnologie japonaise moderne. Shibusawa Keizô suivra cette tendance en se consacrant dans les années 1930 à l'étude des rouleaux peints anciens (Shibusawa 1984) pour élaborer ses corpus «d'objets populaires» (mingu), donnant ainsi naissance aux premiers travaux taxinomiques modernes sur la culture matérielle japonaise.

L'œuvre de Kon se développe parallèlement dans un discours de rupture avec les usages scientifiques du courant auquel il appartient. Le caractère exceptionnel de la catastrophe appelle chez lui une remise en question profonde des dogmes et des présupposés de l'ethnographie japonaise naissante. La chose est particulièrement manifeste dans son intérêt pour le corps. Il refuse d'une part d'inscrire le geste dans un discours de la tradition, tel que le fait Mauss en France, mais tel que le font surtout les folkloristes japonais des années 1920. Son travail est par ailleurs un camouflet à l'autre «ethnologie du corps» pratiquée au Japon, celle de l'anthropologie physique, dont les propos sur les races de l'empire visent essentiellement à légitimer l'entreprise coloniale.

De présenter ici quelques aspects de l'œuvre de Kon Wajirô n'a pas pour seul intérêt de signaler l'existence d'une étude des gestes humains dans un contexte non occidental. Le choix des options retenues par Kon et celles envisagées presque simultanément par Mauss nous permettra également d'interroger la «formule» (Sigaut 2010) dans lequel ce dernier inscrit les techniques du corps.

Différentes manières de porter un bagage (Kon \& Yoshida 1986b : 69)

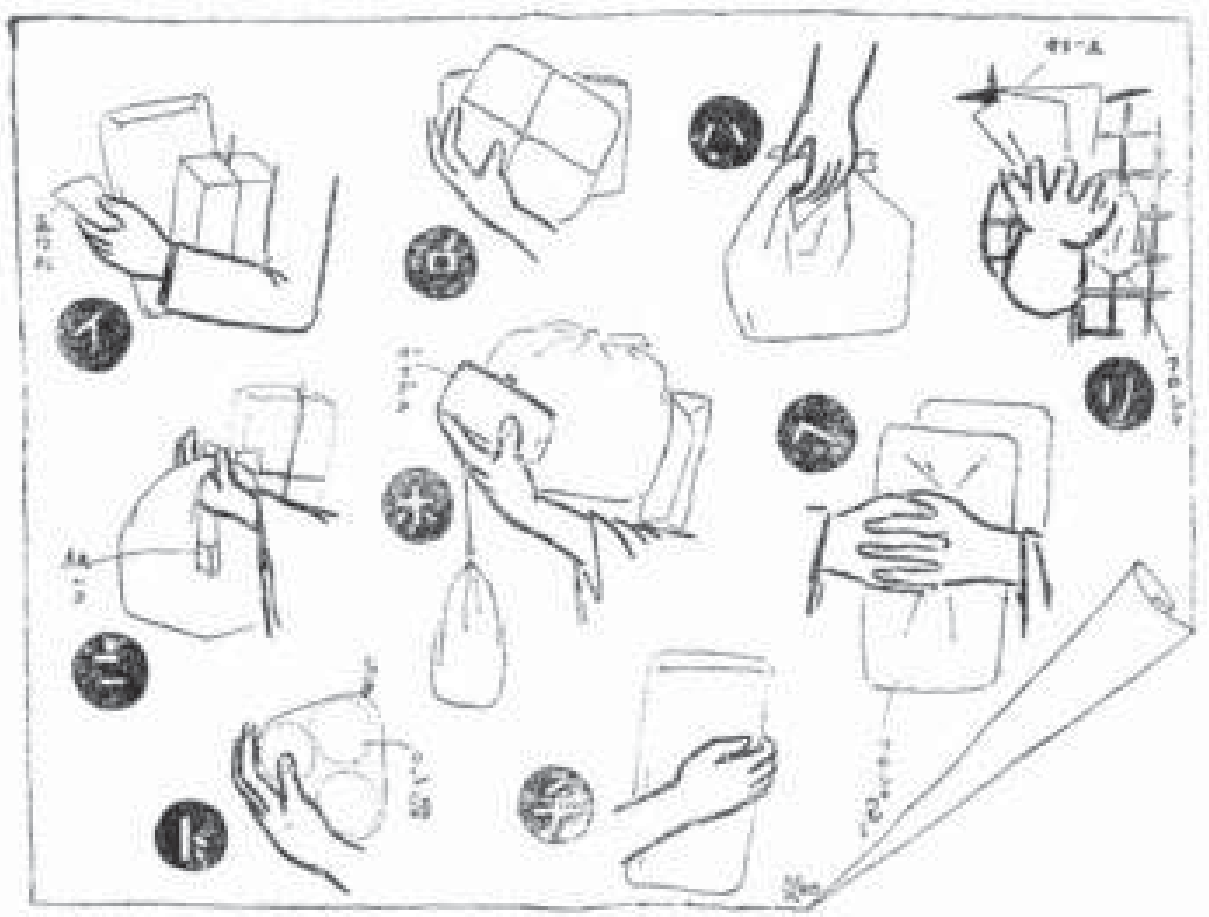




\section{Quelques mots de contexte}

Kon Wajirô se forme d'abord en design ornemental à l'École des Beaux-Arts de Tokyo. Son diplôme obtenu, il est embauché à 24 ans, en 1912, comme assistant au département d'architecture de l'université Waseda où il obtiendra plus tard la charge de professeur.

C'est là qu'en 1916, son directeur, l'architecte Satô Kôichi (1878-1941), l'invite à rejoindre la société Hakubôkai ${ }^{1}$ fondée par ce dernier et le folkloriste Yanagita Kunio. Les membres de ce groupe effectuent des enquêtes à grande échelle portant sur l'habitat des gens du peuple (minka). Le courant initié par Satô et Yanagita, dont Kon est l'assistant direct, se situe à mi-chemin entre un travail d'histoire de l'architecture vernaculaire japonaise et une histoire sociale des pratiques et usages ayant lieu à l'intérieur du foyer. De ces années à la Hakubôkai, Kon publiera en 1922 un livre (Kon 1989) sur les fermes japonaises, faisant toujours référence sur la question.

Cependant, le grand tremblement de terre du Kantô de 1923 fait prendre à Kon une direction radicalement différente de celle du groupe auquel il s'était associé dix ans plus tôt. La méthode encouragée par la Hakubôkai, la quête des survivances et des traditions le laisse insatisfait. Dans les jours qui suivent la catastrophe, il parcourt les ruines de la ville à pied en compagnie d'un ami, le décorateur de théâtre Yoshida Kenkichi (1896-1982). Il est profondément impressionné par l'ingéniosité de la population qui, en collectant débris et matériaux de récupération, rebâtit en autonomie et sans plan directeur logements et échoppes de fortune. Il voit là un terrain plus vivant pour explorer la nature technique de l'habitat japonais que celui qu'offraient les fermes campagnardes, tout en continuant de se consacrer à l'étude des formes de l'architecture vernaculaire.

Le tremblement de terre de 1923 marque donc la rupture définitive avec ses entreprises scientifiques passées. L'intérêt de Kon pour les événements de l'époque va au-delà de la seule inventivité architecturale des habitants de Tokyo. C'est l'ensemble des processus de reconstruction d'une société qu'il peut observer. Ce sont donc ces processus d'étude de la vie ordinaire en période de changement qui vont occuper la seconde moitié de sa carrière, d'abord sous le nom de «modernologie », puis peu de temps après sous le nom « d'archéologie du présent ». La genèse de ces termes est expliquée par Kon lui-même :

« Ce mot archéologie du présent est apparu à l'occasion d'une exposition intitulée Shirabemono qui s'est tenue à la librairie Kinokuniya à l'automne 1927, sur les conseils de son propriétaire Tanabe Moichi avec qui nous partageons des intérêts communs. [...] À cette occasion, comme il semblait peu probable qu'il existe un tel mot dans une langue occidentale, nous nous sommes inspirés du mot kôkogaku [archéologie] qui était une discipline existante. Néanmoins, puisque notre objectif était d'étudier la vie quotidienne telle quelle se déroulait sous nos yeux, nous avons pris l'option gendaigaku [contemporéanologie], en opposition avec l'archéologie qui ne s'intéressait qu’aux questions passées. Cela dit, quitte à créer un mot nouveau, sa version en espéranto nous paraissait plus pertinente, et c'est ainsi que nous l'avons présenté lors de l'exposition » (Kon 1971-1972, vol. 1: 24-25).

Les deux mots sont donc inventés pour l'occasion. Celui de «modernologie » est conçu en espéranto, langage des avant-gardes chez les architectes des années 1920, l'expression

« archéologie du présent » est construite sur le mot « archéologie » (kôkogaku), dans lequel Kon substitue l'idéogramme ( $k 0$ : ancien) par l'idéogramme (gen : présent). 


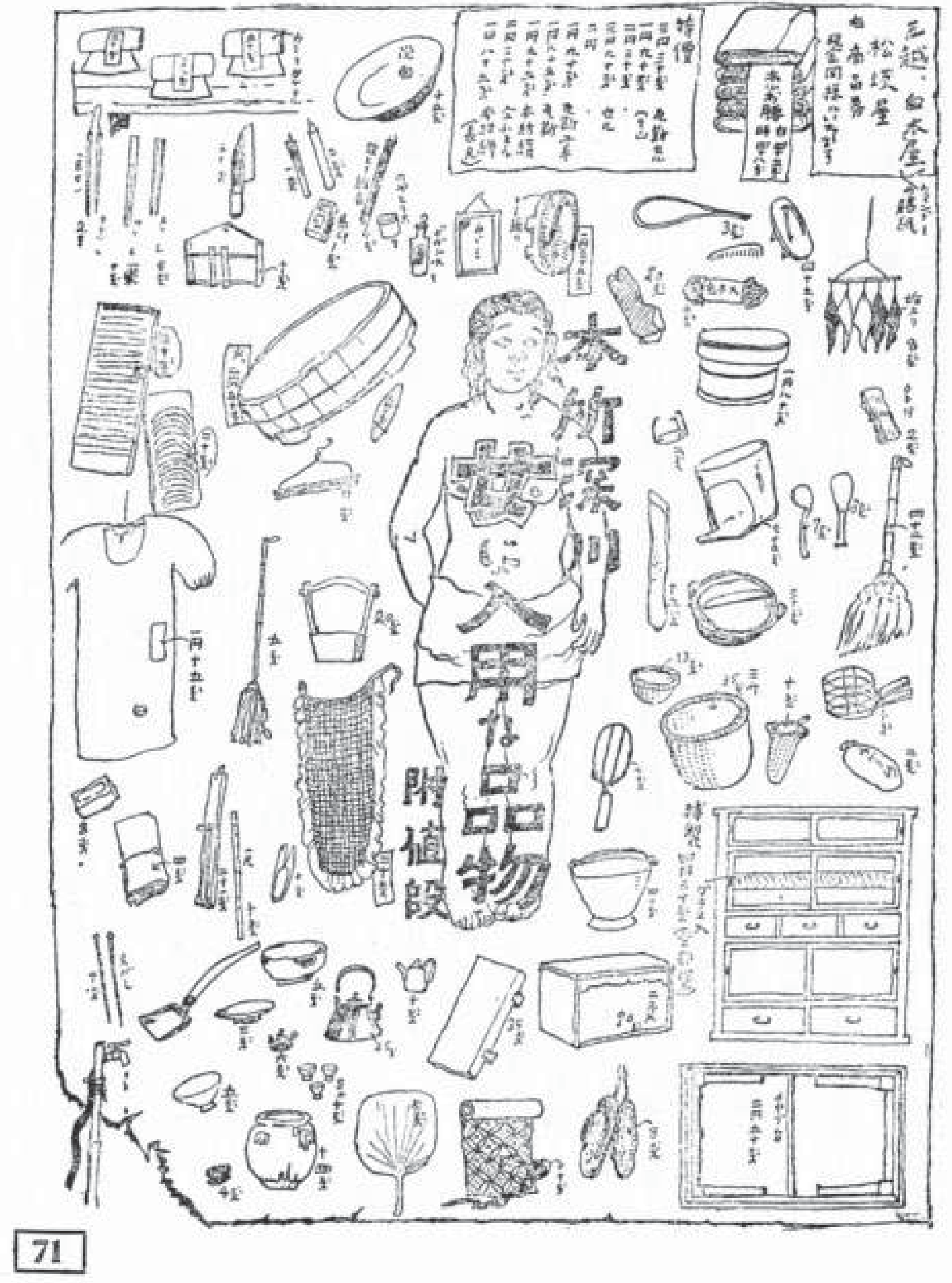

Autour des corps, les biens de première nécessité et leur prix moyens en 1925

Kon Wajirô (Kon \& Yoshida 1986a: 70-71) 


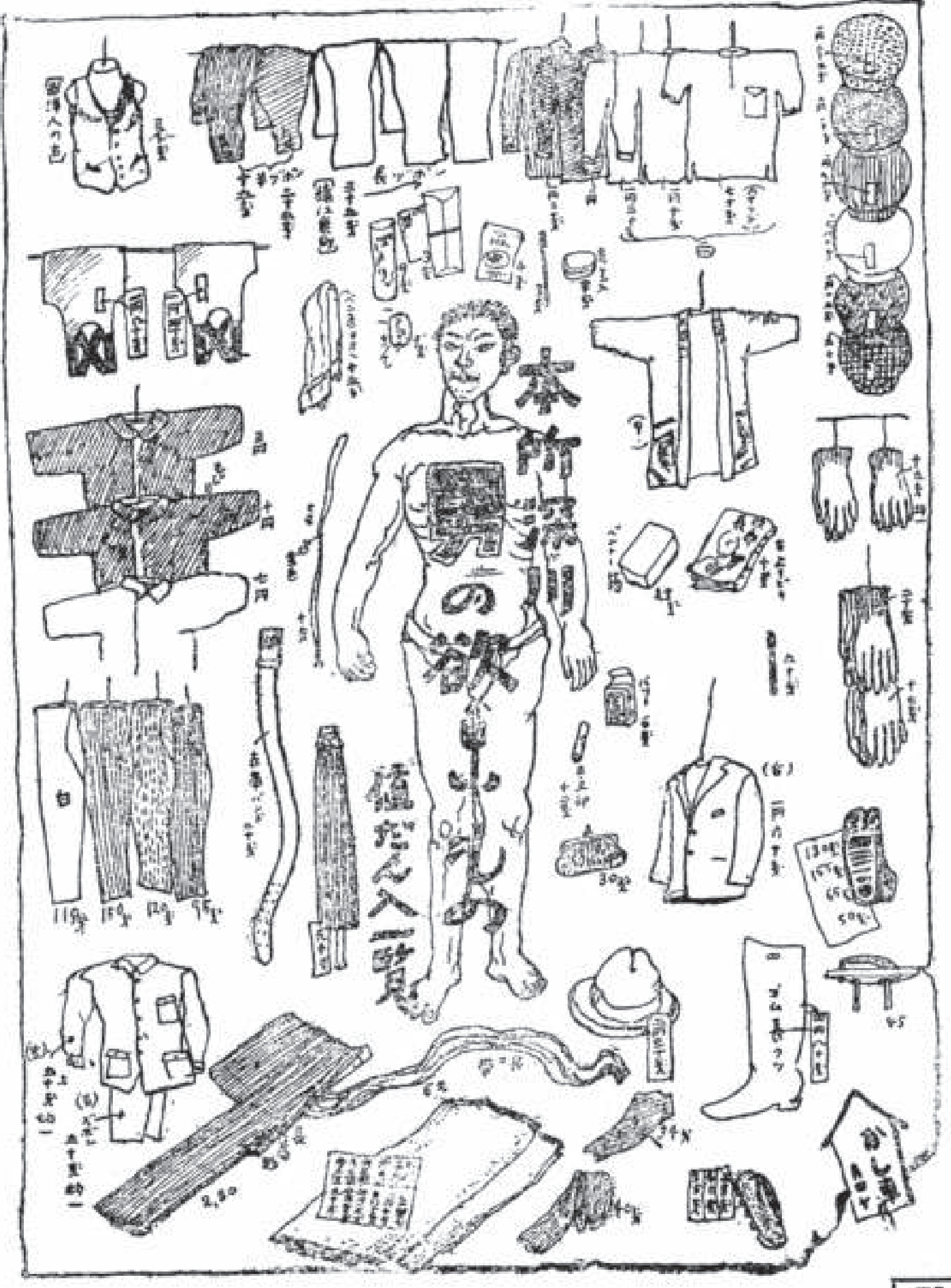


C'est durant les années qui suivent le tremblement de terre du Kantô que Kon publie, en collaboration avec Yoshida Kenkichi, ses plus célèbres ouvrages sur la question : Shirabemono no ten conjointement à l'exposition évoquée ci-dessus, Modernologio : kôgengaku (Kon 1986a) et Kôgengaku saishû (Kon 1986b).

Comme l'a dit Kon dans l'extrait précédent, les deux expressions apparaissent l'une et l'autre en 1927 après quatre années d'enquêtes dans la ville en ruine. Ne sachant quel nom donner à leur discipline, le terme de shirabemono, les « choses à étudier » est d'abord retenu. Puis, pour donner une couleur scientifique à la pratique, et pour prêter à celle-ci un aspect sérieux légitimé par l'idée qu'il pouvait en exister un équivalent occidental, Kon et Yoshida choisissent le mot à consonance étrangère modernologio en lui offrant une traduction japonaise de circonstance, archéologie du présent. La question fut cependant maintes fois posée pendant l'exposition, une telle discipline existait-elle en Occident ? Kon n'en sait rien, et assure le public qu'il changerait le nom de sa méthode dès que la réponse pourrait lui être fournie. Lors d'un voyage aux États-Unis en 1930, il s'enquiert de l'existence d'une « archéologie du présent » à l'étranger. Assuré que rien de tel n'est encore connu de ce côté-là du monde, mais encouragé à poursuivre, il confirme :

«En réalité, je n'ai pas trouvé d'équivalent au terme durant mon séjour en Europe en 1930. J'ai alors présenté notre ouvrage Modernolojio au Prof. Paul H. Nystrom rencontré à l'Université Columbia. Celui-ci menait des recherches sur les modes vestimentaires. Quand je lui ai dit que nous avions baptisé notre discipline la modernologie, il nous a répondu qu'il n'avait jamais rien entendu de tel mais que le terme lui paraissait valide » (Kon 1971-1972, vol. $1: 25$ ).

Ce ne sont pas tant les termes donnés à la pratique de Kon et de Yoshida qui méritent notre attention que le contenu de celle-ci. En effet, la modernologie ou l'archéologie du présent, les deux mots sont donc interchangeables, ont donné naissance à une base de données impressionnante sur les us et pratiques les plus prosaïques du Japon urbain d'avant-guerre, et probablement sans égal dans l'histoire de l'ethnographie. Quelques années avant sa mort, Kon reviendra sur les ambitions de son entreprise:

« La notion de vie quotidienne est extrêmement vague et difficile à saisir. Tel a été le problème que nous avons rencontré quand nous avons choisi d'en étudier la nature.

La vie quotidienne, ce sont tous nos actes, toutes nos expériences entre la naissance et la mort. Téter le sein de notre mère, faire nos premiers pas, jouer, apprendre, manger, s'habiller, habiter, chercher l'amour, travailler, prendre du plaisir, souffrir, dormir, être malade, élever un enfant, entrer en contact avec l'autre, le fait même de vivre, voilà ce qu'on peut appeler la vie quotidienne. [...] Lorsque l'on pose les objectifs de ce que doivent être les études de la vie quotidienne, la question est toujours de savoir ce qu'est cette vie quotidienne et ce qu'on cherche à en dire. » (Kon 1971-72, vol. 5 : 18)

Accompagné de ses étudiants, Kon se poste dans les quartiers de Tokyo et effectue un relevé taxinomique des choses vues, allant des tailles de barbes aux formes des chapeaux, des styles vestimentaires aux manières de fumer, de marcher, de tenir son parapluie, de porter un bagage ou de se reposer en pleine rue. Une autre dimension de cette discipline inventée fut d'étudier dans le détail les possessions matérielles et les habitudes de plusieurs « Tokyoïtes types » au rang desquels les étudiants de Waseda furent les premiers à passer sous la loupe. En visitant leurs domiciles, en les interrogeant sur leurs repas, leurs loisirs, leurs goûts vestimentaires, leurs besoins financiers, la modernologie put établir un profil du Japonais ordinaire des années 1920 avec un soin tout à fait remarquable. 


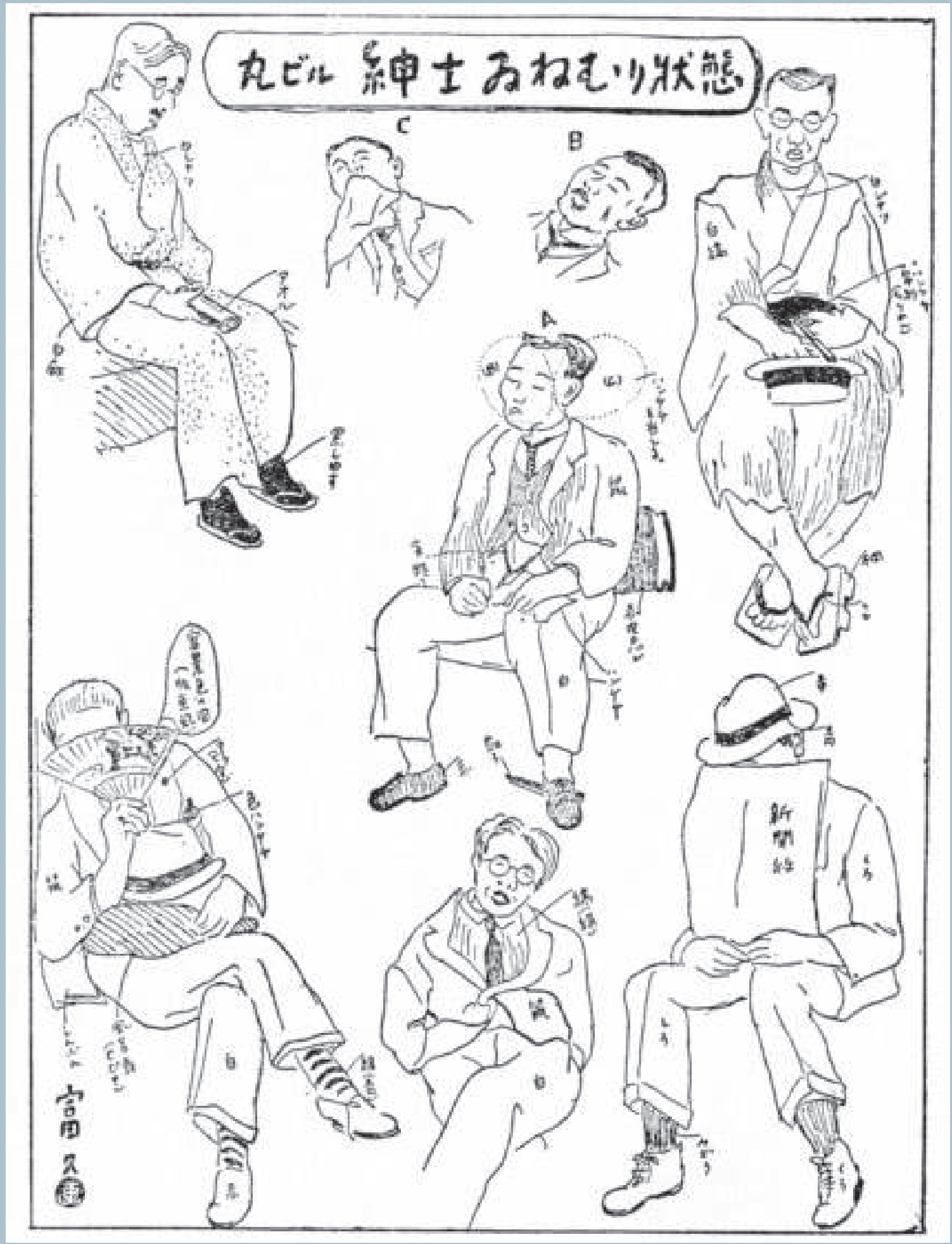

Variations sur l'inemuri (« dormir en étant présent ») observés dans l'immeuble Maru Biru, Tokyo (Kon \& Yoshida 1986a : 244) 
Pour Kon, l'entreprise devait certainement avoir des vertus pédagogiques dans la formation, hétérodoxe il est vrai, de ses étudiants à l'enquête. Les données récoltées serviront certainement aussi, pendant les centaines d'années à venir, à nourrir les travaux des futurs historiens de la société japonaise du $\mathrm{xx}^{\mathrm{e}}$ siècle. Ces informations permettent enfin d'étudier dans un espace donné les changements véritables qui s'opérèrent dans les milieux et les usages sociaux suite à une catastrophe de l'ampleur de celle que Tokyo connut en 1923 et plus généralement dans le processus de modernisation que connut le Japon entre les années 1880 et les années 1920.

Une autre caractéristique de l'œuvre de Kon est celle de l'usage abondant du dessin et son travail est indissociable des planches qu'il a produites ou fait produire durant sa carrière. Le trait de crayon de Kon et le refus de la photographie se distinguent indiscutablement de l'exercice du simple illustrateur scientifique. Fujimori Terunobu voit dans le dessin de l'architecte-enquêteur une influence surréaliste probablement pertinente :

«Alors qu'il menait ses travaux de collecte dans les ruines, Kon s'investit dans une autre entreprise qui prit le nom de Société d’ornementation des baraquements [Barakku sôshokusha]. Avec Yoshida et d'autres de ses camarades rencontrés aux Beaux-Arts, échelle sur l'épaule, pot de peinture à la main et parcourant la ville détruite, il dessina images et motifs sur les devantures des échoppes reconstruites à la hâte. C'est notamment le cas du Café Kirin de Ginza dont il couvrit la façade de vifs coups de pinceau, y dessinant de façon dada des monstres et des motifs primitifs » (Fujimori 1997 : 181).

La pratique purement artistique était donc revendiquée. Ce trait aura très certainement une incidence sur le choix de Kon d'envisager le geste humain selon des paradigmes différents de ceux retenus par Mauss. Voyons comment mettre en balance les deux perspectives.

\section{Lanti-formule de Marcel Mauss}

Dans cette même revue, François Sigaut (2010) s'interrogeait sur la «formule de Mauss», offrant là l'un des rares efforts interprétatifs menés à ce jour au sujet du texte prononcé en 1934. Rappelons la teneur de celle-ci: pour Mauss, l'acte technique n'existe que s'il est traditionnel et efficace. Or, c'est là ce qui nous intéresse, tout en traitant eux aussi du geste humain, les travaux de Kon semblent s'opposer point par point aux présupposés du Français.

La question de la tradition du geste ne mérite pas vraiment que l'on s'épouvante aujourd'hui de l'usage d'un terme qui, d'Éric Hobsbawm (Hobsbawm \& Ranger 1983) à Gérard Lenclud (Lenclud 1987), a depuis fait l'objet de tant de développements. Sigaut souligne pertinemment le problème que pose le mot. Nous admettons comme lui que Mauss ne conçoit le traditionalisme du geste, selon le sens à donner à l'expression dans les années 1930, que dans la mesure où celui-ci est un trait culturel transmis.

Là où Kon, sidéré par la catastrophe de 1923, se distingue de Mauss et de toute l'ethnographie de son temps, c'est qu'il rejette en bloc la notion de tradition qui légitime pourtant les travaux auxquels il avait pris part jusqu'alors. C'est le corps tel qu'il se présente immédiatement qui le préoccupera sa vie durant et dont il étudiera les variations. Le refus de la tradition se fera par exemple en observant les gestes et les déambulations de ces êtres hybrides que sont les modern girls émancipées qui apparaissent dans le contexte 


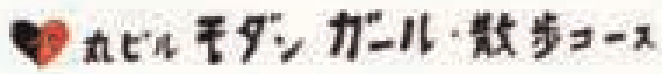

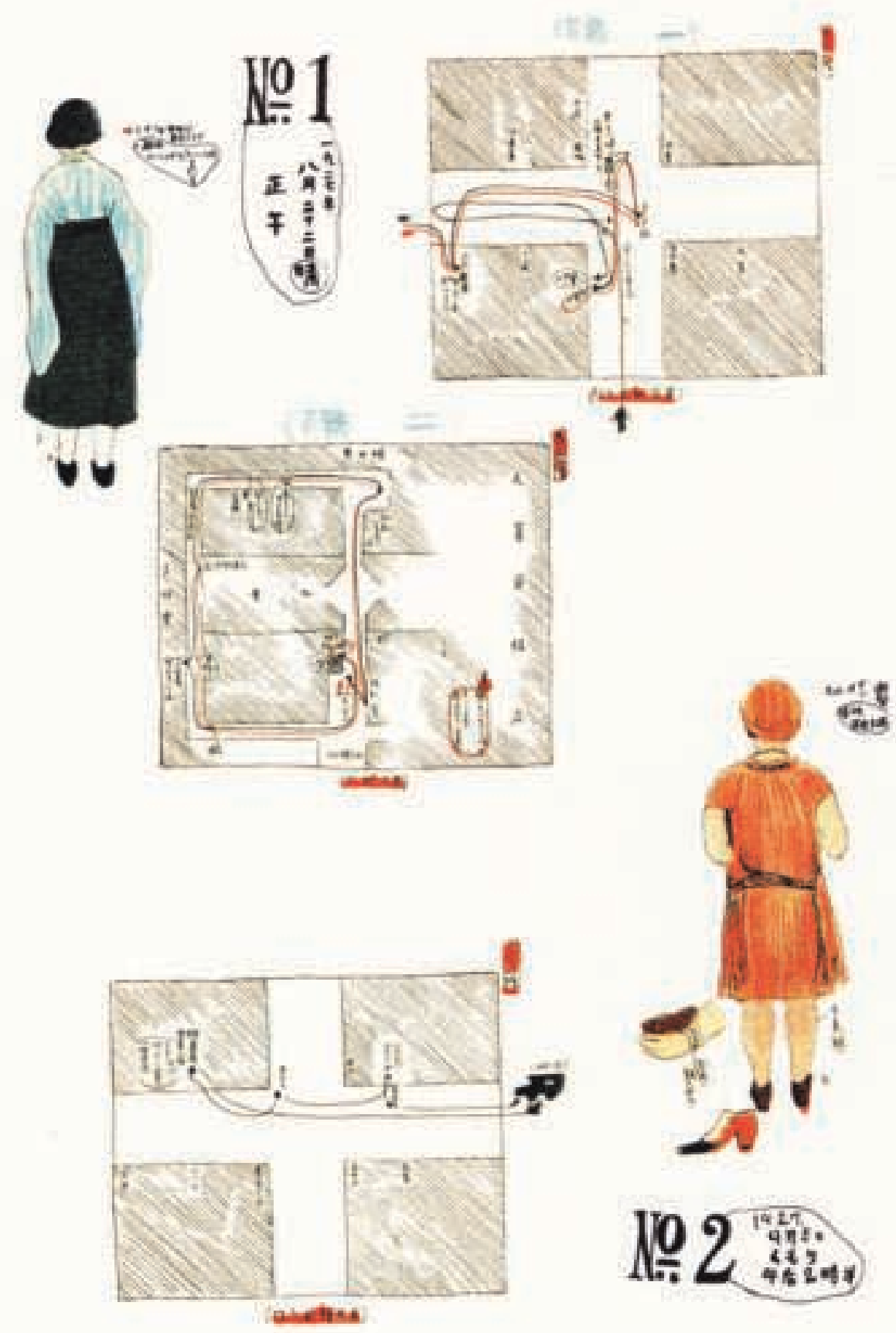

Déambulation de deux modern girls dans l'immeuble Maru Biru, Tokyo (Kon \& Yoshida 1986a : 132) 


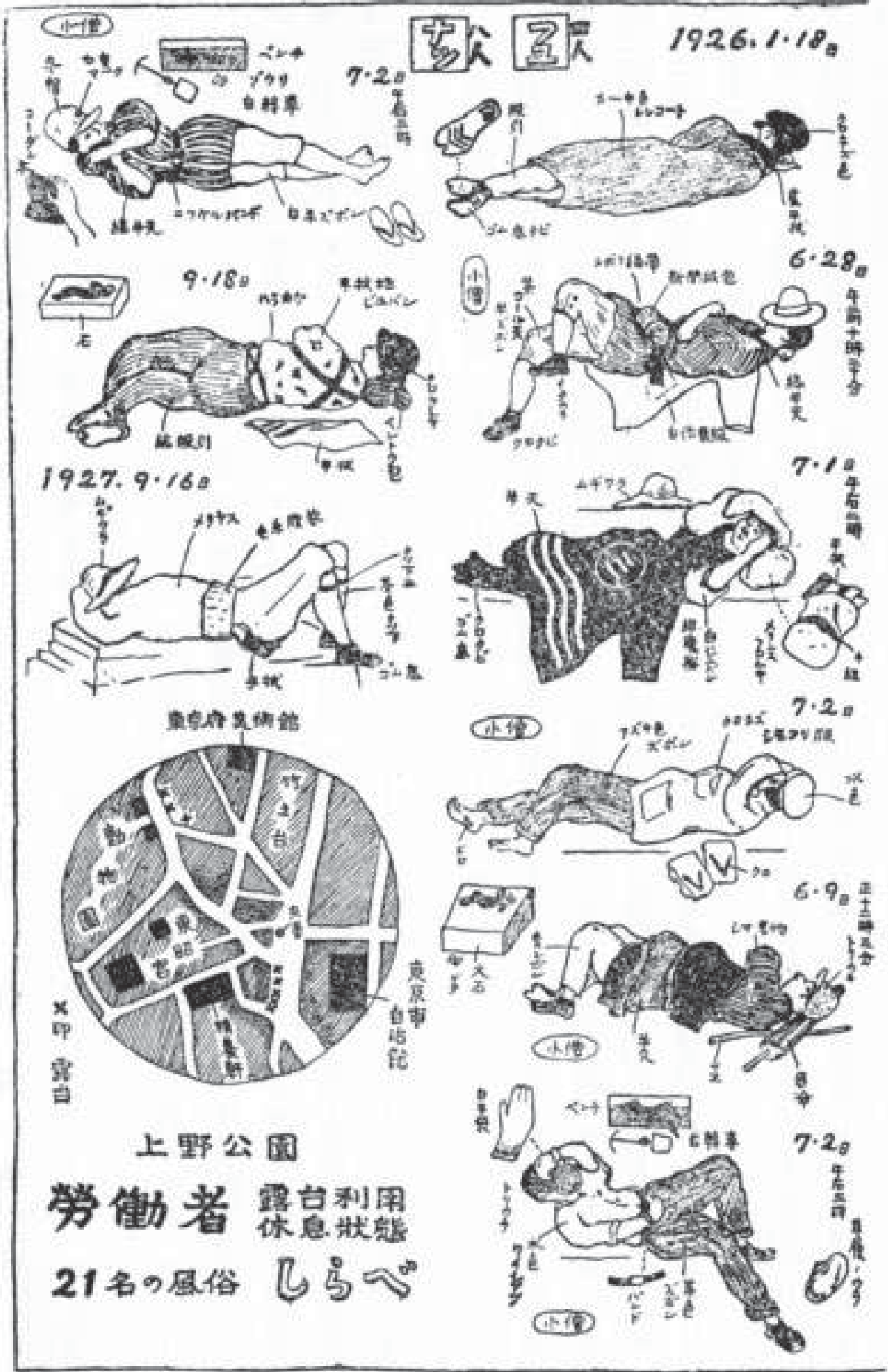



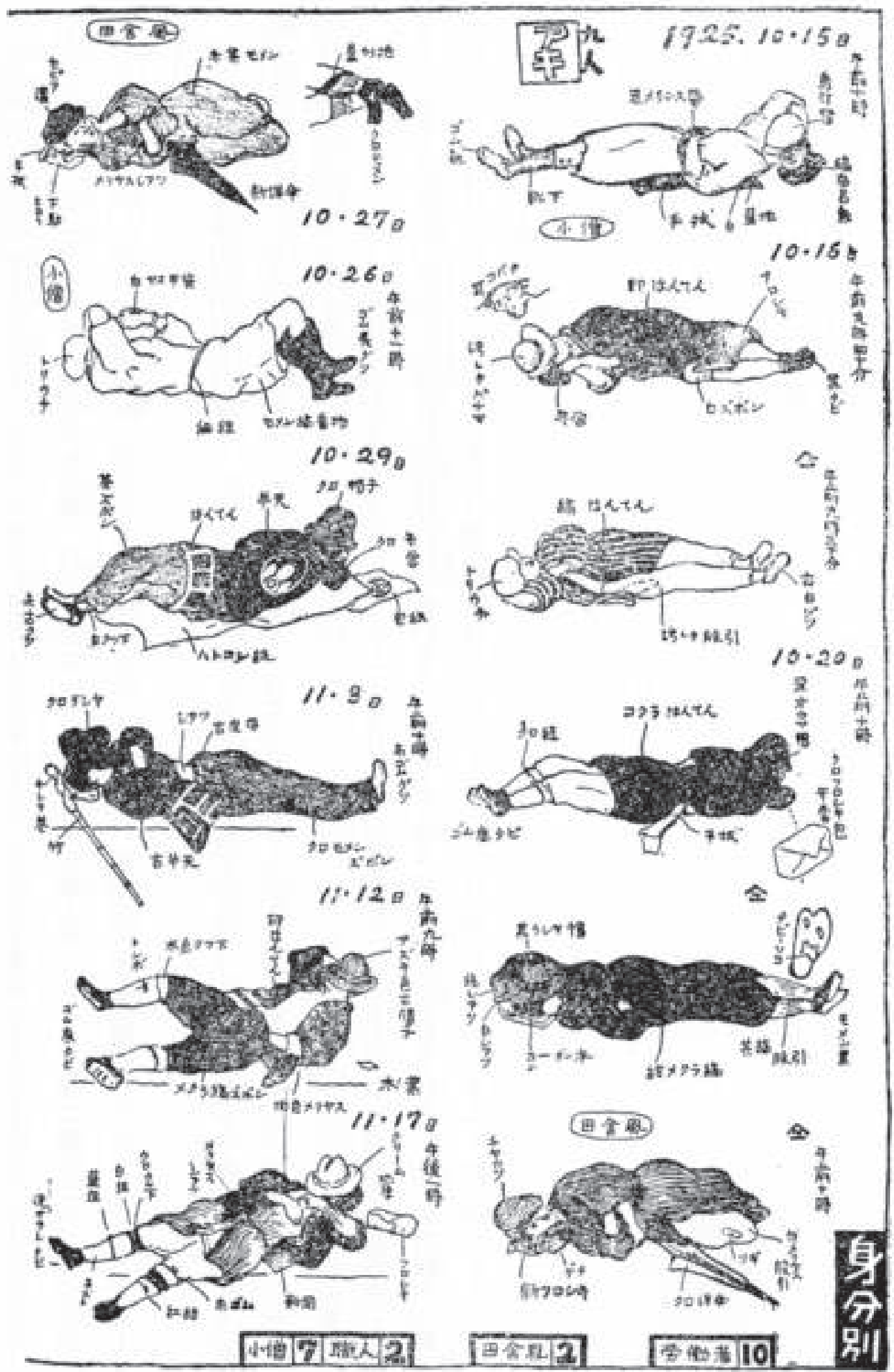
de reconstruction de Tokyo. Jamais un autre ethnologue japonais de l'époque ne s'est intéressé à celles-ci. Mauss relève également l'influence en France des modes de marche américaines, vues au cinéma par les jeunes Parisiennes, mais ne dit rien de la durée nécessaire au transfert culturel pour que la technique du corps devienne véritablement traditionnelle. La «tradition» du geste est-elle un phénomène se déroulant sur le temps long, court, ou est-elle un produit dérivé de l'analyse du geste lui-même (la fameuse tradition inventée)? L'interrogation demeure et Kon, prudemment, ne s'y frotte pas.

Sur la question de l'efficacité du geste ensuite: tout en s'intéressant passionnément au geste, Kon ne voit aucune pertinence à envisager l'efficacité de celui-ci et cela, bien que l'étude des pratiques de construction en situation de désastre doive probablement amener à réfléchir aux plus efficaces manières de bâtir. Le goût pour les avant-gardes artistiques fait ici très certainement prendre au Japonais un chemin différent de celui purement scientifique de Mauss. C'est que Kon est pétri d'influences dada et a une sensibilité évidente pour le geste inefficace, voire absurde. Ainsi, l'étude du geste limité à son caractère efficace présupposé ne l'a jamais préoccupé. Lui-même s'amusait de l'absurdité apparente de sa démarche scientifique tout en la poursuivant avec le plus grand sérieux.

Mauss, de son côté et sans s'en rendre compte, se prend en défaut sur la nature efficace du geste. Pour faire un clin d'œil encore au geste genré des modern girls japonaises et occidentales, citons cette amusante remarque qu'il émet sans plus de précision : « Je ne comprends pas non plus d'ailleurs comment les dames peuvent marcher avec leurs hauts talons » (Mauss 1935 : III, 288). Une chose est certaine, la marche avec de hauts talons n'est probablement pas la marche la plus efficace qui soit. Est-ce le nœud du problème? Ou alors cette marche n'est pas efficace en tant que geste technique de déplacement, mais sans doute comme geste technique de séduction. À ce sujet, Sigaut s'engage dans une analyse nécessaire de la question, dans laquelle il distingue " l'effet » de «l'utilité » du geste, tendant ainsi une main secourable à Mauss pour le sortir de l'embarras dans lequel il s'était mis.

Chez Kon, dont l'ambition est de traiter de tout sans aucune forme de soumission à la théorie, la question de l'efficacité est rejetée puisqu'elle relève pour lui d'un jugement de valeur. Il faut comprendre pour cela son dédain pour les valeurs qui transcendaient l'œuvre des folkloristes et qui, selon lui, les aveuglaient. En quelques mots, juger du geste selon qu'il fût efficace ou non, beau ou non, gracieux ou non (et même traditionnel ou non) ne pouvait l'intéresser.

Ceci nous permet de terminer sur un élément qui ressort de l'exercice de comparaison des travaux de Kon et de ceux de Mauss sur le geste, celui du piège des nomenclatures. En invitant à s'intéresser aux techniques du corps, Mauss établit des embryons de classements intéressants. Il le fait cependant sur la base de gestes que rien ne réunit de manière concrète autrement que par les vocables qui leur sont apposés. En évoquant par exemple les variations de la nage, il présuppose d'un ensemble commun de pratiques du corps glissant sur l'eau. La question mérite pourtant d'être posée. Qu'est-ce qui réunit véritablement ces pratiques sinon le fait qu'on les appelle toutes «nage». Leurs finalités sont-elles réellement les mêmes? Mauss, ni Sigaut d'ailleurs, n'en disent rien. Kon a-t-il été plus performant sur ce point? Rien n'est moins sûr. Cependant, le traitement intellectuel de classification lexical étant par nature différent entre France et Japon, le croisement des approches est riche d'enseignements.

Prenons un exemple que nous allons chercher dans la catégorie maussienne de la «technique de consommation, manger». Ainsi évoque-t-il l'anecdote suivante: 
«Vous vous rappelez l'anecdote du shah de Perse, répétée par Höffding. Le shah, invité de Napoléon III, mangeait avec ses doigts ; l'empereur insiste pour qu'il se serve d'une fourchette d'or. "Vous ne savez pas de quel plaisir vous vous privez", lui répond le Shah » (Mauss 1935 : III, 21).

Certes, Napoléon III et le shah de Perse mangent tous deux. Ils témoignent de variations culturelles transmises dans leurs techniques du manger. Cependant, le rapprochement s'arrête là, car de toute évidence, et Mauss le souligne pourtant, les deux hommes ne mangent pas pour la même raison et peut-être même que ni l'un ni l'autre ne mangent pour s'alimenter. L'un se sert d'une fourchette d'or pour son caractère somptuaire, l'autre mange avec les doigts pour le plaisir. L'un cherche à imposer la civilité de ses mœurs, l'autre s'y refuse pour des motifs culturels. Le seul terme « manger» témoigne ainsi de pratiques fondamentalement différentes et délicates à situer dans une même catégorie typologique.

Lorsque Kon s'intéresse aux repas d'un couple de lithographes et aux gestes qui accompagnent ces repas, il intègre le nombre de cigarettes fumées durant celui-ci. Fumer et manger, deux techniques du corps différentes selon Mauss, et pourtant une finalité probablement similaire dans ces deux gestes, celle de quelques minutes de délassement au cours d'une journée de travail.

Prenons un autre exemple. La dimension du sommeil et de ses variations techniques est abordée par Mauss. Il semble cependant pour lui que l'humain sommeille pour des raisons biologiques et la question du «pourquoi dort-on?» ne se pose pas dans son texte. Chez Kon, le traitement graphique du corps au repos laisse voir deux choses. Une planche illustre les positions de travailleurs manuels endormis en public dans le parc de Ueno à Tokyo (voir précédemment). Une autre planche met en scène des employés de bureau assoupis. Sommeil couché et sommeil assis, dirait Mauss qui souligne légitimement que « la notion que le coucher est quelque chose de naturel est complètement inexacte » (Mauss 1935 : III, 17). Ce n'est pourtant pas la position du corps qui distingue ces deux types de sommeils, mais bien la raison de ce sommeil dont l'étude est inaugurée par Kon.

Dans le cas des travailleurs couchés, le sommeil vise la récupération des facultés physiques après l'effort. Dans le cas des employés assis, le sommeil vise à affirmer la présence du sujet au sein de la communauté. À la suite de ce moment fondateur initié par Kon, au Japon du moins, de l'étude anthropologique du repos, Brigitte Steger, à qui l'on doit plusieurs ouvrages sur les dimensions multiples du sommeil au Japon (Steger 2004, 2007) offrira des explications plus détaillées sur cette forme de sommeil visant à affirmer la présence de l'acteur dans l'espace social. Alors qu'en Occident, le fait de dormir à son poste passe pour un acte répréhensible, il est un acte admis au Japon puisque celui-ci traduit l'investissement du sujet dans son travail au point de s'y consacrer jusqu'à l'épuisement. Associé à l'arrivée matinale sur le lieu d'emploi et au départ tardif de celui-ci, l'endormissement durant l'activité professionnelle est la preuve la plus totale d'un engagement corporel dans son activité. Dormir en étant présent, inemuri en japonais, une technique du corps qui ne se limite pas à la dimension technique qui intéresse Mauss, mais s'inscrit surtout dans sa dimension symbolique dont l'effet, plus que l'utilité, est à isoler analytiquement. La chose est déjà claire chez Kon alors qu'il faudra attendre les travaux de la technologie culturelle des années 1970-1980 en France pour en souligner l'importance.

L'œuvre modernologique de Kon Wajirô peut sembler ubuesque et, à bien des égards, elle l'est. Elle a malgré tout inspiré de nombreux développement depuis, des enquêtes menées par la Société d'observation des rues (Rojô kansatsu gakkai) à l'initiative de l'artiste Akasegawa Genpei (Akasegawa, Fujimori \& Minami 1993) aux travaux de Sakaguchi 
Kyôhei sur les abris de fortune des sans domicile fixes de Tokyo (Sakaguchi 2004). Toutes ces tendances partagent un goût pour le brouillage des frontières entre arts et sciences. Ses résultats sont pourtant probants et ont permis d'identifier, par l'observation, le refus des présupposés théoriques et le choix d'une remise à zéro des compteurs de l'anthropologie, des pratiques corporelles insoupçonnées que l'efficacité et la tradition seules ne sont pas véritablement à même d'expliquer.

\section{NOTES}

Photo d'ouverture: Variations sur les positions du repos. Hokusai, 1819. (Katsushika 2005 : 463)

1. Littéralement, la « Société du chaume blanc », en référence aux toits de chaume des bâtiments étudiés par le groupe.

\section{RÉFÉRENCES}

Akasegawa, G., Fujimori, T. \& S. Minami 1993 Rojô kansatsugaku nyûmon (Introduction à l'étude de l'observation des rues). Tokyo: Chikuma bunko.

Fujimori, T. 1997 «Kon Wajirô » in M. Kano \& S. Tsurumi dir. Minkangaku jiten - jinmeishû (Dictionnaire des études sur les savoirs populaires - volume consacré aux personnes). Tokyo: Sanseidô.

Hobsbawm, E. \& T. Ranger 1983 The Invention of Tradition. Cambridge: Cambridge University Press.

Katsushika, H. 2005 Shozuri Hokusai Manga - Zen (The Complete Hokusai Manga Sketchbooks). Tokyo: Shôgakukan : 463 .

Kon, W. 1971-1972 Kon Wajirô Shû (Euvres de Kon Wajirô), 9 vol. Tokyo : Domesu Shuppan.

Kon, W. 1989 (1922) Nihon no minka (Architecture vernaculaire du Japon). Tokyo : Iwanami bunko.

Kon, W. \& K. Yoshida 1986a [1930] Modernologio / Kôgengaku (Modernologie / archéologie du présent). Tokyo: Gakuyô shobô.

Kon, W. \& K. Yoshida 1986b [1931] Kôgengaku saishû (Recueil d'essais sur l'archéologie du présent). Tokyo : Gakuyô shobô.

Lenclud G. 1987 «La tradition n'est plus ce qu'elle était...», Terrain 9 [En ligne] http://terrain.revues. org/3195.

Mauss, M. 2012 [1935] «Les techniques du corps » in N. Schlanger dir. Techniques, technologie et civilisation. Paris : PUF : $365-394$ [Journal de psychologie normale et pathologique 32 : 271-293].

Sakaguchi, K. 2004 Tôkyô 0-en hausu (La maison tokyoïte à 0 yen). Tokyo : Ritoru moa.

Shibusawa, K. 1984 Shinpan emakimono ni yoru nihon jômin seikatsu ebiki (Études par l'image de la vie quotidienne des gens du peuple dans les rouleaux illustrés, nouvelle édition). Tokyo: Heibonsha.

Sigaut, F. 2010 [2003] « La formule de Mauss », Anthologie raisonnée, TechniquesECulture 54-55 : 357-367. [Efficacité technique, efficacité sociale. TechniquesÉCulture 40].

Steger B. 2004 (Keine) Zeit zum Schlafen? Kulturhistorische und sozialanthropologische Erkundungen japanischer Schlafgewohnheiten, Münster: LIT.

Steger B. 2007 Inemuri. Wie die Japaner schlafen und was wir von ihnen lernen können, Reinbek bei Hamburg: Rowohlt. 


\section{POUR CITER CET ARTICLE}

Kunik, D. 2017 « Gestes et "archéologie du présent" au Japon, des années 1920 à aujourd’hui. Quelques enseignements pour l'ethnologie francophone» in G. Bartholeyns \& F. Joulian, Le corps instrument, Techniques\&Culture 62: 68-83.

\section{RÉSUMÉ}

Gestes et archéologie du présent au Japon, des années 1920 à aujourd'hui

Le tremblement de terre du Kantô de 1923 offre à l'architecte et ethno-folkloriste japonais Kon Wajirô une opportunité radicale de rediscuter les fondements de la démarche disciplinaire qui est la sienne. C'est dans ce contexte qu'il intégrera à sa pratique, qu'il baptise «modernologie», une étude originale des techniques du corps.

\section{ABSTRACT}

Gestures and Japanese Modernology from the 1920's Onwards: Some Lessons for French Anthropology

The 1923 Kantô earthquake urges the Japanese architect and folklorist Kon Wajirô to radically reassess the founding principles of his own discipline. In this context, his research, nicknamed "modernology", integrates an original approach to the study of body techniques.

\section{MOTS CLÉS}

Japon, Kon Wajirô, Marcel Mauss, techniques du corps, modernologie, ethnographie, anthropologie, études folkloriques

\section{KEYWORDS}

Japan, Kon Wajirô, Marcel Mauss, body techniques, modernology, ethnography, anthropology, folklore studies 